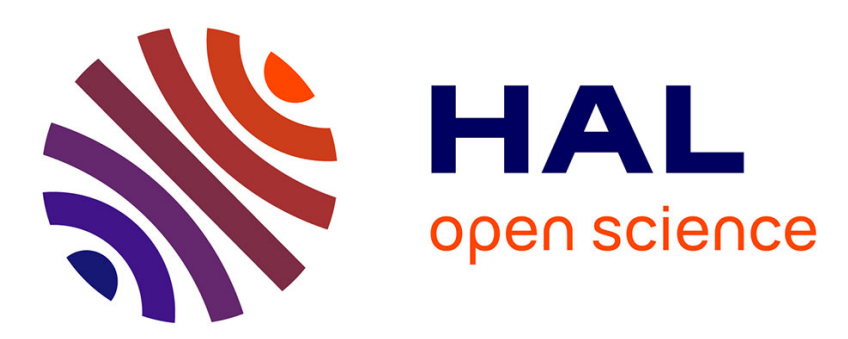

\title{
Complexité cognitive appliquée à la modélisation de l'intérêt narratif
}

\author{
Jean-Louis Dessalles
}

\section{To cite this version:}

Jean-Louis Dessalles. Complexité cognitive appliquée à la modélisation de l'intérêt narratif. Intellectica - La revue de l'Association pour la Recherche sur les sciences de la Cognition (ARCo), 2007, 45 (1), pp.145-165. hal-00616383

\section{HAL Id: hal-00616383 https://hal.science/hal-00616383}

Submitted on 22 Aug 2011

HAL is a multi-disciplinary open access archive for the deposit and dissemination of scientific research documents, whether they are published or not. The documents may come from teaching and research institutions in France or abroad, or from public or private research centers.
L'archive ouverte pluridisciplinaire HAL, est destinée au dépôt et à la diffusion de documents scientifiques de niveau recherche, publiés ou non, émanant des établissements d'enseignement et de recherche français ou étrangers, des laboratoires publics ou privés. 


\title{
Complexité cognitive appliquée à la modélisation de l'intérêt narratif
}

\author{
Jean-Louis DESSALLES*
}

Resume. Nous définissons la complexité cognitive comme une notion dérivée de la complexité de Kolmogorov. Nous montrons qu'une partie importante de ce qui retient l'intérêt des êtres humains, notamment lors de la sélection des événements spontanément signalés ou rapportés, peut être prédite par un saut de complexité cognitive. Nous évaluons les conséquences de ce modèle pour l'étude de la pertinence conversationnelle.

Mots clés : complexité, pertinence, intérêt, probabilité, narration.

Abstract. Cognitive Complexity as a Criterion for Modeling Narrative Interest. Cognitive complexity is defined as a special case of Kolmogorov complexity. This notion is claimed to be essential for predicting which states of affairs appear interesting to a human mind, as evidenced by the selection of events that are spontaneously signaled or reported: interesting events correspond to a cognitive complexity drop. We consider the consequences of this model for the study of conversational relevance.

Key words: complexity, relevance, interest, probability, narrative.

\section{INTRODUCTION : MODELISER L'INTERET NARRATIF}

L'intérêt narratif constitue un chapitre central de l'étude de la cognition humaine, et l'on ne peut que s'étonner du fait qu'il n'ait pas fait l'objet de davantage de travaux. De nombreuses études ont porté sur la modélisation de l'attention sélective, notamment visuelle (voir Itti et Koch, 2001 pour une revue). En revanche, les mécanismes qui mobilisent l'intérêt pour des situations complexes sont moins bien compris. L'enjeu pour la modélisation de la communication humaine est de comprendre ce qui pousse les individus à signaler de manière quasi réflexe certaines situations plutôt que d'autres, qu'il s'agisse de situations immédiatement ou récemment perçues ou de situations tirées de la mémoire épisodique à long terme.

L'activité qui consiste à signaler ou rapporter des événements occupe une part considérable du temps humain. Si l'on considère que le temps de conversation représente un cinquième du temps d'éveil (Dunbar, 1998) et que les narrations peuvent constituer la moitié de ce temps de parole, nous passons un dixième de notre temps à communiquer des événements dignes d'intérêt (voir par exemple Eggins et Slade, 1997, p. 265 pour une estimation typique, sachant que la variance est très importante selon les conversations). Le comportement narratif est aussi digne d'intérêt parce qu'il semble uniquement humain. Dans notre espèce, il apparaît très tôt : les enfants de moins d'un an transforment leur curiosité en acte de communication en cherchant activement à attirer l'attention

*ENST (LTCI, UMR 5141), ParisTech, 46 rue Barrault - 75013 Paris, dessalles@enst.fr, www.enst.fr/ jld. (C) 2007 Association pour la Recherche Cognitive. 
d'un parent vers un stimulus inhabituel, comme un pantin dansant (Carpenter et al., 1998 ; Liszkowski et al., 2004). Inversement, au même âge, les enfants humains comprennent l'intention communicative d'un geste de pointage (Behne et al., 2005), ce qui n'est pas le cas chez le chimpanzé, même adulte (Call et al., 1998).

Certaines tentatives pour inventorier les facteurs de l'intérêt ont été menées dans des travaux classiques de la théorie du journalisme (MacDougall et Reid, 1957 ; Galtung et Ruge, 1965). La récence, la proximité, le caractère exceptionnel ou négatif des événements et le nombre d'individus affectés ont une influence décisive sur la valeur de l'information (newsworthiness). La proximité au sens large (géographique, politique, émotionnelle) a particulièrement été mise en avant (Maclean et Pinna, 1958). Le problème de ce type d'inventaire des facteurs qui jouent sur l'intérêt narratif est lié à leur hétérogénéité. Certains critères, comme la présence de caractéristiques économiques, politiques, géographiques ou culturelles, ou la référence à des personnes de l'élite, constituent une catégorisation de l'événement, alors que d'autres, comme la simplicité, la proximité, le pathos, l'aspect conflictuel, l'opportunité ou la familiarité, peuvent être évalués sur n'importe quelle situation. D'autres facteurs parfois mentionnés, comme le caractère significatif (meaningfulness), la pertinence ou l'importance (significance), introduisent une confusion avec ce qu'il s'agit d'expliquer. Une autre difficulté vient de ce que les études en théorie du journalisme portent sur la dernière phase de sélection des événements, au niveau de la salle de rédaction. Elles portent donc sur des nouvelles déjà sélectionnées, par exemple par des agences de presse, si bien que des facteurs aussi importants que le caractère conflictuel, l'opportunité, la proximité, l'importance et la familiarité, pris ensemble, n'expliquent que $20 \%$ de cette sélection finale (Berkowitz, 1990).

Un moyen indirect d'évaluer les facteurs qui jouent sur l'intérêt narratif consiste à observer les paramètres qui influent sur la mémorisation des événements. Les individus mémorisent plus efficacement les comportements qui paraissent incohérents ou qui s'écartent des stéréotypes (Stangor et McMillan, 1992). Les tests de rappel d'histoires montrent également que la discrimination mémorielle est meilleure pour les actions atypiques que pour les actions typiques (Woll et Graesser, 1982 ; Shapiro et Fox, 2002). Les circonstances les plus exceptionnelles peuvent entraîner une mémorisation précise et à très long terme de la situation, comportant même le rappel de détails non pertinents (Brown et Kulik, 1977).

L'image qui semble se dégager est qu'une multitude de facteurs sans liens entre eux influent sur la saillance et sur l'intérêt (Landragin, 2004). Il est permis de se demander s'il est possible d'aller au-delà du simple catalogage et si une théorie cognitive de l'intérêt narratif pourra voir le jour. L'objet de cet article est de promouvoir cette direction, en proposant des éléments qui pourraient servir de base à une telle théorie.

\section{INTERET ET PROBABILITE}

Dans des travaux antérieurs (Dessalles, 2002 ; 2005), nous avons souligné l'importance de la probabilité dans l'intérêt conversationnel des événements. La notion d'information que Claude Shannon a définie dans un tout autre contexte (Shannon, 1948) se trouve être un bon prédicteur de l'intérêt des événements. L'intérêt $I$ d'un événement de probabilité $p$ s'écrit ainsi :

$$
I=\log _{2} 1 / p
$$


L'ensemble des prédictions que cette formule permet de faire est impressionnante. Mentionnons-en quelques-unes.

\subsection{Atypicité}

L'intérêt croît de manière prévisible avec le caractère atypique de l'événement. On s'intéressera par exemple à une saisie record de drogue par les douanes si celle-ci dépasse de beaucoup ce à quoi on est habitué. Lorsque l'on possède une intuition de la distribution de la classe d'événements, par exemple une distribution normale d'écart-type $\sigma$, on peut montrer que l'intérêt d'une situation à $k$ écarts-types de la moyenne, tel qu'il est défini par la formule (1), croît selon une fonction $A(k)$ qui varie comme $k^{2}$.

\subsection{Rareté}

Les événements rares retiennent systématiquement l'attention des individus qui peuvent mesurer cette rareté. Ce sera le cas, par exemple, pour la chute d'une météorite. Cet intérêt se traduit directement en probabilité par la formule (1).

\subsection{Proximité}

La proximité égocentrique des événements rares joue un rôle décisif sur leur intérêt. Ainsi, la chute d'une météorite est beaucoup plus intéressante si elle se produit à un kilomètre que si elle se produit à $1000 \mathrm{~km}$. La formule (1) peut prédire cet effet de manière quantitative. En appliquant une loi de Poisson à la distribution de l'événement rare, on peut montrer que l'intérêt décroît comme le double du logarithme de la distance spatiale (si l'on se place à deux dimensions). Plus généralement, l'influence de la distance se traduit par la formule :

$$
I=-\log _{2}\left(D_{v} v_{e}\right)
$$

$D_{v}$ représente la densité hypervolumique de la classe d'événements, et mesure donc sa rareté. L'hypervolume considéré intègre diverses variables pertinentes, comme l'espace physique, le temps, la proximité sociale, etc. La variable $v_{e}$ représente le plus petit hypervolume égocentré contenant l'événement observé. On vérifie que plus ce volume est faible, plus l'intérêt est élevé. Cette relation prédit l'effet de récence, bien connu en journalisme : « News is a perishable product, good only when fresh » (Warren, 1934, p. 15). L'intérêt décroit, selon (2), comme le logarithme de l'ancienneté de l'événement. La formule (2) permet également de prédire l'effet mort-kilomètre, selon lequel un accident, pour susciter un intérêt égal, doit impliquer davantage de victimes s'il se produit en un lieu plus reculé (Dessalles, 2005).

\subsection{Banalisation}

L'un des comportements remarquables que l'on peut observer dans les conversations sur le mode narratif consiste à banaliser l'événement qui est rapporté par d'autres (Dessalles, 2006b). Ainsi, dans un extrait où la première locutrice raconte avoir vu des arbres aux racines gigantesques dans un arboretum d'Hawaï, la seconde locutrice mentionne qu'elle a vu le même type d'arbre au Vietnam. L'effet de banalisation est obtenu, dans le cadre de la formule (1), en signalant que l'on alloue une valeur supérieure à la probabilité 
$p$. En mentionnant un événement analogue, l'interlocuteur peut, selon les cas, influer sur $D_{v}$ ou sur l'écart-type $\sigma$ de la distribution. En mentionnant un élément de connaissance qui augmente la plausibilité de l'événement initial, il peut changer la probabilité a posteriori de l'événement. La mécanique de la banalisation, bien prédite par (1), conduit au phénomène remarquable des tournois narratifs (Tannen, 1984, p. 100).

\section{DIFFICULTES DE L'APPROCHE PROBABILISTE}

Malgré son indéniable efficacité dans la prédiction de l'intérêt narratif, l'approche probabiliste, ou shannonienne, rencontre un certain nombre de difficultés qui nous ont amené à la replacer dans un cadre plus général et plus satisfaisant sur le plan de la modélisation cognitive. La plus évidente de ces difficultés concerne son incapacité à rendre compte de l'intérêt des situations exceptionnelles, qui sont pourtant extrêmement fréquentes dans les conversations spontanées.

\subsection{Exceptions et coïncidences}

Le fait de rencontrer deux religieuses en train de faire un jogging constitue un événement intéressant pour ceux qui peuvent mesurer l'incongruité de ce comportement. Même si la formule (1) a quelque chose à dire sur la contribution de la proximité spatiale et temporelle de l'événement, elle ne peut mesurer son caractère atypique. Les interlocuteurs n'ont pas besoin d'avoir accès à des statistiques sur les activités sportives des personnes religieuses, car ils ne mesurent pas l'intérêt de la situation par la déviation en nombre d'écarts-types de la moyenne. La notion de probabilité intègre mal la notion d'incongruité ou d'exceptionnalité. Considérons l'exemple suivant, rapporté par N. Norrick (Norrick, 2000, pp. 55-56, détails de transcription omis).

Brianne: It was just about two weeks ago. And then we did some figure drawing. Everyone was kind of like, "oh my God, we can't believe

$[\ldots]$ it." We- y'know, Midwest College, y'know,

Brianne: like a ... nude models and stuff. And it was really weird, because then, like, just last week, we went downtown one night to see a movie, and we were sitting in [a restaurant], like downtown, waiting for our movie, and we saw her in the [restaurant], and it was like, "that's our model" (laughing) in clothes

Addie: (laughs) Oh my God.

Brianne: we were like "oh wow." It was really weird. But it was her. (laughs)

Addie: Ohno. Weird.

Brianne: I mean, that's weird when you run into somebody in Chicago.

Addie: yeah.

Certains aspects de cet extrait sont bien prédits par la formule (1) : les mentions «it was just about two weeks ago » et «just last week» jouent sur la proximité temporelle. La précision finale concernant le lieu, Chicago, est essentielle car elle fournit un accès à la faible densité $D_{v}$ de l'événement, qui aurait été bien moins intéressant, parce que plus probable, dans une petite ville. 
En revanche, la formule (1) n'inclut pas l'influence du décalage initial : être amenée à peindre un modèle nu dans un Midwest College. Elle ne mesure pas non plus l'importance du décalage entre les deux aspects successifs explicitement mentionnés du personnage, d'abord nue puis « in clothes». Il est facile de jouer sur ces paramètres et d'observer leur impact sur l'intérêt : l'histoire aurait été moins bonne si peindre un nu avait été une activité normale pour la locutrice, ou si le modèle avait été initialement habillé.

Cet exemple peut être vu comme un cas particulier de coïncidence. Les événements qui relatent une coïncidence ont un effet puissant sur l'attention des interlocuteurs. Pourtant, dans bien des cas, la formule (1) se révèle incapable de le prédire. Considérons l'exemple suivant (Le Figaro, 20 mars 2004)

Surprise, suspense et perplexité pour les gendarmes maritimes de Nice et les policiers du commissariat d'Antibes confrontés à une énigme peu banale: celle de la découverte dans la baie des Anges, dans l'après midi, des corps flottants entre deux eaux de deux femmes d'âge mûr aux élégantes tenues vestimentaires.

[...]

En fait, une extraordinaire coïncidence aurait poussé deux femmes d'âge similaire, mais ne se connaissant absolument pas, à choisir le même jour, de rester très élégantes pour aller en pleine matinée se noyer presque simultanément au même endroit.

Plusieurs paramètres de la coïncidence ont un effet graduel fondamental sur l'intérêt: le fait que les deux suicides se soient produits à quelques kilomètres de distance, presque à la même heure. Ici, les paramètres de distance spatiale et temporelle ne sont pas égocentriques, mais allocentriques : ils portent sur les deux termes de la coïncidence. La ressemblance entre les deux situations est, elle aussi, essentielle pour l'intérêt de l'histoire, ce que la formule (1) n'est pas non plus en mesure d'expliquer: le fait qu'il s'agisse dans les deux cas de femmes d'âge mûr, élégantes, parées de leurs bijoux, qui ont choisi de se noyer en descendant dans la mer au petit matin. Toute altération de cette ressemblance nuit à l'intérêt. Inversement, tout autre élément commun quoique fortuit, comme le fait de porter le même prénom, aurait accru l'intérêt de l'histoire. Peut-on l'expliquer?

\subsection{Effets de structure}

Un autre aspect de l'intérêt spontané humain que la théorie des probabilités peine à expliquer vient de l'existence de structures remarquables. Ainsi, la configuration 66666 du compteur kilométrique provoquera immanquablement l'excitation d'un enfant qui la remarque et qui, de ce fait, cherchera à attirer l'attention de la conductrice. Même si une telle configuration est intuitivement improbable, la théorie lui alloue la même probabilité qu'une configuration apparemment inintéressante comme 67246. Un tel phénomène a été classiquement analysé comme une 'erreur' cognitive : un biais de représentativité (Kahneman et Tversky, 1972). L'importance fondamentale des phénomènes de structure pour la compréhension de ce qui fonde l'intérêt humain va cependant apparaître dans la suite. 


\subsection{Le manque de fondements cognitif des probabilités}

Il y a plus grave. Pour constituer un modèle cognitivement plausible du jugement d'intérêt narratif, on ne doit faire appel qu'à des notions accessibles à la cognition des individus. Or, ce n'est certainement pas le cas de la notion de probabilité qui entre dans la formule (1). De nombreux auteurs ont étudié le divorce entre les probabilités mathématiques et l'intuition apparemment biaisée que les sujets humains peuvent en avoir. Citons l'erreur dite du parieur (gambler fallacy) (Terrell, 1994), l'erreur de conjonction (conjunction fallacy) (Tversky et Kahneman, 1983), l'erreur du taux de base (base-rate fallacy) (Bar-Hillel, 1980) ou la primauté donnée aux informations concrètes et proches, associée à la négligence des informations de fréquence (Nisbett et Ross, 1980).

Toutefois, le problème n'est pas seulement que le comportement humain cadre mal avec les prédictions mathématiques. Il vient également de ce qu'à l'inverse, la théorie classique des probabilités est incapable de fournir un cadre acceptable pour rendre compte des capacités cognitives en matière de jugement probabiliste (Dessalles, 2006a). La principale raison vient de ce que les différentes approches mathématiques de la probabilité (à l'exception notable de celle de Ray Solomonoff (Solomonoff, 1997)) présupposent la connaissance explicite de l'ensemble des alternatives. On peut accepter que le jeu de dés soit suffisamment conventionnel pour que chacun sache ignorer la position précise des dés sur la table ou la température de la pièce comme non pertinents. L'ensemble $\Omega$ des alternatives se résume ainsi à $\{1,2,3,4,5,6\}^{n}$ où $n$ est le nombre de dés. En revanche, lorsque l'on est confronté à des religieuses en train de faire leur jogging, il est plus délicat de délimiter $\Omega$. S'agit-il de l'ensemble des couples de religieuses, de l'ensemble des joggers, de l'ensemble des situations de rencontre en jogging ? Si $\Omega$ dépend de la sélection des paramètres pertinents, personne ne dit à l'observateur que la couleur de leurs yeux peut être ignorée, alors que l'âge des deux femmes et la présence du chapelet qui ballote au rythme de la course peuvent contribuer à renforcer l'intérêt de l'histoire.

Dans ce qui suit, nous proposons une autre approche de l'intérêt, qui présente le double intérêt d'englober les résultats acquis avec la formule (1) et de constituer un modèle acceptable sur le plan cognitif.

\section{LA NOTION D'INATTENDU}

Un moyen plus fondamental d'analyser la part de l'intérêt mesurée par la formule (1) est de la fonder sur la notion d'inattendu (unexpectedness), définie comme la différence entre la complexité cognitive attendue $C_{\text {exp }}$ de la situation considérée $s$ et sa complexité observée $C_{o b s}$ :

$$
U(s)=C_{e x p}(s)-C_{o b s}(s)
$$

La complexité cognitive d'une structure est définie, par analogie avec le concept de complexité de Kolmogorov (Li et Vitanyi, 1993) : la taille minimale du "programme » qui permet de caractériser la structure considérée. Cette notion dépend donc du modèle cognitif sous-jacent dont on dispose, et de la complexité des opérations élémentaires qu'il met en jeu.

Si nous reprenons l'exemple du compteur kilométrique, nous comprenons pourquoi la configuration 66666 est plus inattendue que 67246. Le programme cognitif minimal qui construit cette dernière consiste à «copier » un chiffre 
non instancié en cinq exemplaires, puis à réaliser cinq instanciations indépendantes. Cette complexité attendue est bien supérieure à la complexité observée dans la première configuration. Il existe en effet un programme bien plus simple pour produire 66666, qui consiste en l'instanciation d'un seul chiffre décimal suivie d'une copie en cinq exemplaires. La différence de complexité $U$ égale donc 4 fois la complexité d'une instanciation. Cette dernière se mesure comme le nombre de bits nécessaires à cette instanciation, soit :

$$
U=4 \times \log _{2} 10
$$

Pour faire le lien avec la formule (1), il suffit de redéfinir la probabilité selon :

$$
p=2^{-U}
$$

Cette formule donne une probabilité de $10^{-4}$ d'observer la configuration 66666, ce qui correspond à la probabilité classique de l'événement où tous les digits sont identiques, sans que leur valeur soit précisée.

On notera que $U$ est par définition une grandeur positive, ce qui est conforme avec l'exigence que la probabilité définie par (4) est inférieure à 1 . La complexité d'observation, dans le cas le plus défavorable, est celle du programme attendu, si bien que $U$ est alors nul. C'est ce qui se passe dans les situations de la vie courante auxquelles nous n'accordons pas d'intérêt puisqu'elles se conforment à nos attentes.

L'intérêt de la notion d'inattendu dépasse largement son lien avec la probabilité, si bien que la formule (4) peut être considérée comme définitoire de la probabilité à partir de l'inattendu, et non l'inverse (Dessalles, 2006a). Pour le montrer, nous allons réinterpréter les phénomènes de dépendance de l'intérêt vis-à-vis de l'atypicité, de la proximité et des coïncidences, avant de considérer d'autres classes de phénomènes inaccessibles à l'analyse probabiliste.

\section{COMPLEXITE CONCEPTUELLE ET INTERET NARRATIF}

Examinons les conséquences naturelles de la définition (3) en nous concentrant ici sur la caractérisation conceptuelle de la situation rapportée. Dans ce nombreux cas, le sujet qui estime l'intérêt d'une situation dispose d'une expérience sous la forme d'un prototype. Dans notre exemple des religieuses, il aura accès à un prototype de nonne qui, vraisemblablement, la présentera comme ayant un comportement posé. La complexité conceptuelle $C^{\text {conc }}(s)$ de la situation $s$, pour ce sujet, peut s'écrire comme la somme de deux complexités, liées respectivement à la caractérisation du prototype de référence $r$ et à la caractérisation de la situation $s$ considérée au sein de son prototype.

$$
C^{\text {conc }}(s)=C(r)+C(s \mid r)
$$

Nous allons considérer deux cas, selon que la complexité conceptuelle est liée à une opération de discrimination par rapport au prototype ou qu'elle s'appuie sur une structure remarquable.

\subsection{Complexité de discrimination}

Le plus souvent, la caractérisation conceptuelle d'une situation consiste en une discrimination :

$$
C^{\text {conc }}(s)=C(r)+C^{\text {discr }}(s \mid r)
$$

Dans le calcul de la complexité attendue $\left(C_{\text {exp }}\right), C^{\text {discr }}(s \mid r)$ a une valeur généralement élevée, qui vaut au maximum $C^{\text {discr }}(s \mid r)=\log _{2} N$, où $N$ est le nombre 
d'instances dans le prototype. Dans le calcul de la complexité observée $C_{o b s}$, la discrimination repose généralement sur l'observation d'une caractéristique particulière $F$, ce qui permet d'écrire, pour la complexité observée $\left(C_{o b s}\right)$, $C^{d i s c r}(s \mid r)=C(r)+C(F)+C^{d i s c r}(s \mid r \& F) .{ }^{1}$ Dans le cas des religieuses en train de faire leur jogging, $F$ est le fait de courir. Si l'on compare nos nonnes sportives à des nonnes standard, ou plus généralement la situation caractérisée par $F$ avec la situation normale, le caractère inattendu dû à la discrimination par $F$ vaut :

$$
U_{\text {discr }}(s \mid r)=C^{\text {discr }}(s \mid r)-C^{\text {discr }}(s \mid r \& F)-C(F)
$$

Cette formule permet de prendre en compte l'intérêt des situations exceptionnelles. Si le fait de courir rend les deux religieuses de notre exemple uniques, alors $C^{d i s c r}(s \mid r \& F)$ est nul.

$$
U_{\text {discr }}(s \mid r)=C^{\text {discr }}(s \mid r)-C(F)
$$

Il en résulte une prédiction qui n'existait pas dans le cas probabiliste : une caractéristique simple est plus intéressante qu'une caractéristique complexe. Il aurait été moins intéressant de raconter que l'on a croisé une religieuse dont le chapelet comportait un nombre incorrect de perles. Cette caractéristique, tout en conférant une caractérisation suffisante pour rendre la situation rare, voire unique, aurait grevé par trop le terme $C(F)$ pour la plupart des auditeurs, au point de paraître parfaitement ennuyeuse.

La formule (7) permet de rendre compte de l'intérêt des situations atypiques. Reprenons l'exemple de la saisie de drogue. Si $N$ est le nombre d'événements de ce type dont on peut supposer l'existence, alors l'information nécessaire pour caractériser l'un d'entre eux est $C^{d i s c r}(s \mid r)=$ $\log _{2} N$. Si cette saisie est d'une ampleur $F$ atypique à $k$ écarts-types de probabilité $p$, la caractérisation s'opère sur en moyenne seulement $N p$ situations, ce qui nécessite une complexité $C^{\text {discr }}(s \mid r \& F)=\log _{2} N-A(k)$ bits d'information, avec $A(k)=\log _{2} 1 / p$. Noter que la probabilité $p$ n'est ici qu'un intermédiaire de calcul, dans la mesure où l'on suppose que les sujets ont un accès direct, grâce à leur expérience, à la fonction $A(k)$. Dans le cas gaussien, comme nous l'avons mentionné, $A(k)$ varie comme $k^{2}$. L'inattendu d'une telle situation atypique est donc donné par:

$$
U_{\text {discr }}(s \mid r)=A(k)-C(F)
$$

Là encore, la simplicité de la caractéristique $F$ prise en compte est essentielle, ce que le modèle shannonien ne pouvait pas prévoir. Ceci explique que certains événements ne puissent intéresser que des spécialistes. Les composants mandibulaires qui permettent à Stylogymnusa subantarctica, un scarabée des îles Auckland, de sucer et de percer tout à la fois, le rendent unique. Cette unicité peut passionner un entomologiste qui trouvera la caractérisation simple, mais elle risque d'ennuyer la plupart des novices en la matière.

L'inattendu de discrimination regroupe tous les cas de déviance. Une part considérable des narrations conversationnelles portent sur des situations déviantes que sont les meurtres, les actes barbares, ou plus communément les actes socialement inappropriés (c'est également le cas des narrations fictives, que nous ne considérons pas ici). Ces formes de déviance, si l'on omet l'aspect

\footnotetext{
${ }^{1}$ En toute rigueur, le deuxième terme est $C(F \mid r)$ : il mesure la complexité de $F$ sachant que le prototype de référence $r$ est donné. Nous supposons ici que la connaissance de $r$ n'influe pas sur cette complexité, de manière à alléger l'écriture.
} 
émotionnel qui peut leur être attaché et qui doit être traité séparément (Dessalles, 2007), offrent un caractère fortement inattendu en raison de la simplicité de leur discrimination. La déviance repose toujours sur une caractéristique $F$ qui rend la situation unique ou rare dans sont type, soit de manière qualitative (formule (8)), soit de manière quantitative (formule (9)).

\subsection{Complexité structurelle}

La formule (5) offre une autre possibilité pour l'inattendu conceptuel. Il suffit que la structure de la situation observée soit plus simple qu'attendu. L'exemple du compteur kilométrique en offre une parfaite illustration. En tant que kilométrage, 66666 est beaucoup plus simple que 67246 et est donc beaucoup plus intéressant.

L'estimation de la complexité structurelle dépend crucialement du modèle cognitif auquel on se réfère. Pour fixer les idées, nous pouvons nous placer dans le cadre défini par Michael Leyton $(1992 ; 2001)$ : toute structure apparaît comme résultant d'une opération de transfert obtenue par l'action d'un groupe sur un autre groupe (voir Dessalles, 2006a). La complexité de l'opération résultante se calcule alors, de manière récursive, en ajoutant les complexités de ces deux groupes considérés eux-mêmes comme des structures. Ainsi, la configuration 66666 est produite par le transfert d'un chiffre instancié par un groupe de translations. Sa complexité est celle d'une instanciation plus celle d'une copie (Dessalles, 2006a).

Une nouvelle comme l'occurrence d'un tirage de loto consécutif, comme 17-18-19-20-21-22 intéressera un nombre considérable de joueurs, même parmi ceux qui n'ont pas participé à ce tirage. La complexité observée est celle d'une instanciation, soit $\log _{2} 17$ puisque la complexité d'un entier $n$ est en général de l'ordre de $\log n$ (Li et Vitanyi, 1993), plus celle du groupe $(\mathbf{Z},+)$ lié à l'addition. La nouvelle aurait été moins intéressante pour un tirage comme 17-20-23-26-29-32 en raison de la complexité supérieure du groupe $(3 \mathbf{Z},+)$. En revanche, elle aurait été plus intéressante si la série avait commencé en 1, en raison de la simplicité des premiers chiffres. Si, lors du premier tirage du Loto en France, le 19 mai 1976, la combinaison 1-2-3-4-5-6 avait été produite par l'appareil, bien peu auraient invoqué l'équiprobabilité des tirages pour ne pas s'en étonner. Par la formule (4), l'improbabilité, voire l'étonnement, mais aussi l'intérêt, résultent d'une simplicité inattendue. Un tirage consécutif produit une diminution de complexité de près de 28 bits, et est donc très inattendu. Noter que cette ampleur d'inattendu n'est bien mesurée que par ceux qui ont une expérience de la complexité habituelle des tirages, ce qui n'est pas forcément le cas pour ceux qui n'ont jamais accordé d'attention à ce jeu. Les joueurs habituels, en revanche, en viennent à considérer qu'un tirage consécutif est strictement impossible (Savoie et Ladouceur, 1995 ; Dessalles, 2006a).

\section{COMPLEXITE D'ACCES ET INTERET NARRATIF}

Les événements qui retiennent l'intérêt des êtres humains sont des états de choses situés, c'est-à-dire qu'ils peuvent être caractérisés non seulement conceptuellement, comme nous venons de le voir, mais également dans l'espace, dans le temps et par les individus qu'ils impliquent. Cette caractérisation spatiale, temporelle, sociale, etc. ajoute à la complexité de caractérisation conceptuelle. 


\subsection{Inattendu et proximité}

Nous définissons la complexité d'accès par :

$$
C^{a c c}(s)=\log _{2}\left(v_{e} / v_{s}\right)
$$

Comme précédemment, $v_{e}$ désigne le plus petit hypervolume égocentré qui contient la situation cible $s$. Le facteur $v_{s}$ représente le plus petit hypervolume centré sur $s$ et englobant $s$. Dans l'exemple des nonnes en train de courir, la projection spatiale de $v_{s}$ est un disque de quelques mètres, propre à englober la scène. Sa projection temporelle est de quelques secondes, la durée d'observation de la scène. La complexité attendue est le nombre de bits nécessaires pour caractériser la localisation de l'événement dans son aire de réalisation habituelle. Si $D_{v}$ est la densité hypervolumique du type d'événement, la complexité attendue pour accéder à la localisation est donc $C_{\text {exp }}(s)=\log \left(V_{e} / v_{s}\right)$ où $V_{e}=1 / D_{v}$ est le volume égocentré moyen où l'on attend une instance. La complexité observée $C_{o b s}(s)=\log \left(v_{e} / v_{s}\right)$ mesure quant à elle la quantité de bits effectivement nécessaire pour caractériser la localisation de l'événement observé. L'inattendu d'accès est donné par la différence entre ces deux complexités, conformément à la définition (3).

$$
U_{a c c}(s)=-\log \left(D_{v} v_{e}\right)
$$

Cette formule, identique à (2), est obtenue par un mode de calcul radicalement différent, qui permet de faire davantage de prédictions. On retrouve l'influence des proximités spatiale, temporelle, sociale ou autre sur l'intérêt, par l'intermédiaire de $v_{e}$ qui doit être aussi petit que possible.

La formule (11), appliquée dans le domaine de la proximité sociale, permet d'expliquer certaines préférences dans la communication humaine, comme le fait que l'on s'intéresse plus volontiers à des histoires qui arrivent à des gens qu'on connaît, des proches ou des célébrités. Cela s'explique par le fait que l'accès cognitif à ces personnes est moins complexe. Cette préférence produit un phénomène bien connu dans l'analyse des médias, lié à une rétroaction positive : plus on parle d'une personne, plus elle devient cognitivement simple; ce qui lui arrive devient donc plus intéressant et offre d'autres occasions de parler de cette personne.

\subsection{Points de repère}

La formule (10) permet de prédire l'utilisation que les auteurs de narrations conversationnelles font de points de repère. Elle permet même de prédire quels repères seront utilisés. Par exemple, pour raconter à mes interlocuteurs un incendie dont j'ai été témoin, je repère l'endroit, une école primaire, par rapport à mon lieu de travail, puis par rapport à la rue de l'Espérance toute proche, puis par rapport au salon de coiffure qui fait le coin. Le choix de ces repères successifs est essentiel pour conserver une faible valeur à $C_{o b s}(s)$. Si je cherche à situer $s$ directement à partir du lieu d'énonciation, j'obtiens une valeur significative qui nuit à l'intérêt. L'histoire est entendue comme: "l'incendie a eu lieu à une vingtaine de kilomètres d'ici. » L'utilisation de points de repère permet de faire chuter $C_{o b s}(s)$ selon la formule suivante qui est un développement de la formule (10) :

$$
C^{a c c}(s)=C(L)+\log \left(v_{L} / v_{s}\right)
$$

La détermination du repère $L$ (landmark) requiert une complexité $C(L)$. L'hypervolume $v_{L}$ est le plus petit hypervolume centré sur $L$ qui contient la 
cible $s$. Cette formule contraint le choix de $L$ de manière à rendre $C^{a c c}(s)$ minimale :

$$
L=\operatorname{argmin}\left(C(L)+\log \left(v_{L} / v_{s}\right)\right)
$$

On constate que la possibilité d'utiliser un repère de faible complexité est propre à augmenter l'intérêt. Ainsi, un incendie mineur au quatrième étage de la Tour Eiffel le soir du 22 juillet 2003 fit l'objet d'une information dans les journaux nationaux: la facilité d'accès cognitif au repère, la Tour Eiffel, confère une très faible valeur à $C(L)$, si bien que l'événement est aussi intéressant pour un lecteur Français que s'il s'était produit dans le voisinage immédiat de son domicile. La formule (12) peut s'appliquer de manière récursive, ce qui prédit l'emploi de plusieurs repères successifs. Dans le cas de $n+1$ repères, cela donne :

$$
C^{a c c}(s)=C\left(L_{0}\right)+\sum C^{a c c}\left(L_{i} \mid L_{i-1}\right)+\log \left(v_{L_{n}} / v_{s}\right)
$$

Chaque terme $C^{a c c}\left(L_{i} \mid L_{i-1}\right)=\log \left(v_{L_{i-1}} / v_{L_{i}}^{0}\right)$ résulte de l'application de (12) en considérant que $v_{L_{i-1}}$ est l'hypervolume centré sur $L_{i-1}$ qui contient $L_{i}$ et $v_{L_{i}}^{0}$ correspond à l'hypervolume occupé par $L_{i}$. Le jeu de repères qui maximise l'intérêt est celui qui minimise $C^{a c c}(s)$. Cette formule prédit que de nouveaux repères seront insérés dans la description tant que cela permet de diminuer la valeur de $C^{a c c}(s)$. Le choix des repères résulte donc d'un calcul cognitif élaboré, qui tient compte de la complexité d'accès du point de vue de l'interlocuteur. Ainsi, pour raconter l'incendie de l'école primaire, je peux prendre le quartier de la Butte aux Cailles comme repère si je m'adresse à un habitant de la région parisienne, tandis qu'une description plus complexe sera requise pour un interlocuteur connaissant mal Paris. Ceci contribue à expliquer le fait que certaines histoires ne puissent intéresser que certains interlocuteurs, ceux pour lesquels la baisse de complexité de la formule (3) sera suffisamment apparente. Le même constat s'applique lorsque la distance est sociale et que les repères sont des personnes, comme lorsque l'on raconte une anecdote à propos de la voiture du frère de Paul, selon que l'interlocuteur connait Paul ou ne le connaît pas. ${ }^{2}$ Ce phénomène explique en partie la raison pour laquelle les nouvelles portent préférentiellement sur des personnes déjà connues du public.

\subsection{Coïncidences}

La notion de complexité d'accès fournit une explication élégante de l'impact des coïncidences sur l'intérêt. L'idée est que la complexité chute, conformément à la formule (3), car la description du double événement est plus simple que la somme des complexités de chaque événement. Raisonnons dans un premier temps dans l'hypothèse où les deux événements qui constituent la coïncidence sont exactement de même nature : par exemple deux chutes de météorites. La complexité attendue est deux fois la complexité attendue pour l'accès à l'un des deux événements : $C_{\text {exp }}(s)=2 \times C^{a c c}\left(s_{1}\right)=2 \times \log \left(V_{e} / v_{s}\right)$, où $V_{e}=1 / D_{v}$ est l'hypervolume égocentré moyen où l'on s'attend à constater une instance de l'événement. La complexité observée est obtenue en considérant que l'on accède au deuxième événement à partir du premier : $C_{o b s}(s)=C^{a c c}\left(s_{1}\right)+$ $C^{a c c}\left(s_{2} \mid s_{1}\right)$, où cette fois $C^{a c c}\left(s_{1}\right)=\log \left(v_{e} / v_{s}\right)$ est la complexité d'accès observée pour l'un des événements. La complexité d'accès relative $C^{a c c}\left(s_{2} \mid s_{1}\right)=$

\footnotetext{
${ }^{2}$ La « distance » sociale à prendre en compte ne doit pas être confondue avec le plus court chemin dans un graphe, la première variant généralement exponentiellement en fonction du second, sauf en présence de fortes contraintes spatiales (Csányi et Szendröi, 2004).
} 
$\log \left(v_{a} / v_{s}\right)$ dépend de l'hypervolume allocentrique $v_{a}$ centré sur le premier événement et englobant le second. On obtient donc que, dans le cas d'une coïncidence, l'inattendu d'accès vaut, par différence entre les complexités attendue et observée :

$$
U_{a c c}(s)=-\log \left(D_{v}^{2} v_{e} v_{a}\right)
$$

La formule (15) nous permet de rendre compte du rôle crucial des distances allocentriques, que le modèle shannonien laissait inexpliqué. Comme nous l'avions observé dans l'exemple des deux noyées de la Baie des Anges, il est essentiel que les deux événements se soient produits à proximité spatiale/temporelle/sociale l'un de l'autre, de manière à minimiser $v_{a}$. La présence des autres facteurs de la formule (15) nous rappelle que la coïncidence sera d'autant plus intéressante qu'elle concerne un événement rare ( $D_{v}$ faible) et qu'elle est proche et récente ( $v_{e}$ faible).

Si l'on cesse de supposer que les événements sont identiques, on s'éloigne encore davantage de toute explication en termes probabilistes. En revanche, le principe de saut de complexité continue à s'appliquer, comme nous allons le vérifier.

\section{INDIVIDUALISATION COGNITIVE}

La thèse centrale défendue ici est que les individus s'intéressent à des situations qui sont plus simples à individualiser que ce à quoi ils s'attendent. L'individualisation d'une scène comme la rencontre avec un chat demande une description minimale de taille significative : il faut discriminer le chat de tous les autres chats, et isoler le lieu et l'époque de la scène avec suffisamment de précision. La tâche d'individualisation est plus aisée si le chat possède quatre oreilles ou pèse $10 \mathrm{~kg}$, et si la scène de rencontre s'est produite il y a cinq minutes à proximité.

La complexité qui intervient dans la formule (3), qu'elle soit attendue ou observée, est relative à l'individualisation de la situation considérée. Nous nous intéressons bien entendu à l'individualisation en tant qu'opération cognitive, telle qu'elle peut être réalisée par les êtres humains dans leur vie quotidienne. Il ne s'agit en aucun cas de rechercher une caractérisation «objective» de la complexité des situations. Les mesures de complexité données par les formules (8), (9) et (11) dépendent crucialement de l'expérience de l'individu par le choix de la référence $r$ et de la densité $D_{v}$. De plus, la formule (11) n'a de sens que pour un point de vue égocentré. Pour être prédictif, c'est-à-dire pour rendre compte du comportement des individus, le modèle doit précisément éviter toute utilisation de notion «objective » à laquelle les individus n'auraient pas accès.

Compte tenu du rôle cognitif particulier que jouent certains paramètres graduels comme l'espace, le temps et la proximité sociale, il est intéressant de scinder l'opération d'individualisation : un état de choses devient un événement si (1) il peut être individualisé conceptuellement et (2) il peut être situé, i.e. être individualisé par instanciation dans l'espace, dans le temps, par les personnes impliquées, etc. La complexité de cette double individualisation s'écrit :

$$
C(s)=C^{c o n c}(s)+C^{a c c}(s)
$$

Dans cette formule, $C^{\text {conc }}(s)$ représente la complexité d'individualisation conceptuelle, et $C^{a c c}(s)$ représente la complexité d'accès. Nous avons étudié les paramètres qui influent sur la première (formules (5) à (9)) et sur la seconde 
(formules (10) à (15)). Le tableau 1 récapitule les différentes notions sur lesquels porte le calcul de la complexité lors de l'individualisation cognitive.

$C(s)$

$C^{\text {conc }}(s)$

$C^{\text {discr }}(s \mid r)$

$C(F)$

$C^{a c c}(s)$

$$
D_{v}^{r}
$$

$v_{e}$

hypervolume (selon les dimensions spatiales, temporelle, sociale...) égocentré minimal contenant la situation.

$v_{a} \quad$ hypervolume allocentré minimal centré sur un élément d'une coïncidence contenant l'autre élément.

Tableau 1. Récapitulatif des principales notions utilisées dans le calcul de la complexité cognitive.

L'application de la formule (3) conduit à distinguer deux sources d'inattendu :

$$
U(s)=U_{\text {conc }}(s)+U_{a c c}(s)
$$

Cette formule montre le partage de la tâche d'individualisation entre l'individualisation conceptuelle et l'individualisation de localisation. Ces deux sources d'inattendu, et donc d'intérêt, s'observent particulièrement bien dans l'exemple des deux noyées de la Baie des Anges. Nous avons vu comment la proximité spatiale et temporelle conférait une valeur élevée à $U_{a c c}(s)$ par l'intermédiaire de la formule (15). Maintenant, nous pouvons intégrer l'influence sur l'intérêt de la proximité conceptuelle entre les deux noyades. La complexité d'individualisation conceptuelle attendue est deux fois celle d'une noyade standard: $C^{\text {conc }}(s)=2 \times C^{\text {conc }}\left(s_{1}\right)$. La complexité conceptuelle observée peut s'écrire: $C^{\text {conc }}(s)=C^{\text {conc }}\left(s_{1}\right)+C^{\text {conc }}\left(s_{2} \mid s_{1}\right)$, où $C^{\text {conc }}\left(s_{2} \mid s_{1}\right)$ est la complexité liée au fait d'individualiser conceptuellement la situation $s_{2}$ sachant qu'on l'a déjà fait pour $s_{1}$. Plus l'analogie de structure entre les deux situations est forte, et plus cette complexité est faible, puisqu'il suffit de conceptualiser les diffé- 
rences. ${ }^{3}$ D'où l'importance, dans la narration sur la double noyade, d'insister sur le fait qu'il s'agissait de deux femmes, toutes deux d'âge mûr, et toutes deux élégamment vêtues. En termes leytoniens (Leyton, 2001), l'histoire est d'autant plus intéressante que le transfert entre les deux noyades est important, consistant essentiellement dans le groupe de symétrie qui envoie l'une sur l'autre. Comme pour le kilométrage remarquable, plus l'instanciation (âge, tenue, ... ) est grande avant le transfert, plus le saut de complexité est important.

Le caractère prédictif de la formule (17) ne doit pas être sous-estimé. En particulier, cette formule donne un nouvel éclairage sur les détails qui sont inclus dans une narration, ainsi que l'insistance et l'exagération qui peuvent moduler ces informations (Tannen 1989, p. 141). Les informations propres à augmenter la différence de complexité cognitive pour l'interlocuteur peuvent et doivent être mentionnées. La formule prédit également que toute mention qui n'affecte pas la différence de complexité sera reçue comme superflue, voire incongrue. ${ }^{4}$ Ainsi, dans la narration concernant le modèle nu, Brianne ne fait pas mention du fait que la personne en question était brune ou que son prénom commençait par la lettre $\mathrm{N}$.

\section{COMPLEXITE ET NARRATION}

De manière assez générale, la complexité d'individualisation conceptuelle s'obtient par l'intermédiaire d'une situation de référence $r$, selon la formule (6). Si l'on combine avec la formule (16), on peut écrire : $C(s)=C(r)+$ $C^{d i s c r}(s \mid r)+C^{a c c}(s \mid r)$, si l'individualisation conceptuelle par rapport au prototype prend la forme d'une discrimination. Or $C(r)$ peut être à son tour décomposé selon la formule $(16): C(s)=C^{c o n c}(r)+C^{a c c}(r)+C^{\text {discr }}(s \mid r)+C^{a c c}(s \mid r)$. Généralement, les descriptions narratives localisent le prototype, si bien que $C^{a c c}(s \mid r)$ est nul. Ainsi, dans l'exemple des religieuses, la localisation porte sur la rencontre avec des religieuses, avant toute précision concernant leur attitude. En d'autres termes, dans ces descriptions médiatisées par un prototype, l'opération d'accès mesurée par $C^{a c c}$ n'est généralement effectuée qu'une seule fois. ${ }^{5} \mathrm{Si}$ l'on écrit maintenant l'inattendu associé à $s$ en appliquant la précédente équation aussi bien à la complexité attendue qu'à la complexité observée, on obtient, dans la mesure où le prototype ne produit lui-même aucun inattendu conceptuel :

$$
U(s)=U_{\text {discr }}(s \mid r)+U_{a c c}(r)
$$

$U_{a c c}(r)$ constitue une écriture commode qui représente une différence de complexité d'accès à la situation, considérée comme son prototype. Dans le cas où le prototype est fréquent, $U_{a c c}(r)$ peut être négatif. Le découpage exprimé dans la formule (18) revient dans ce cas à transférer une part supérieure à $U(s)$ dans le premier terme. Le passage par un prototype de référence $r$ n'est accep

\footnotetext{
${ }^{3}$ Il s'agit là d'une propriété cognitive fondamentale des analogies. Une bonne analogie est celle qui minimise la complexité cognitive globale du couple de situations considéré. La solution d'une équation analogique à quatre termes : « $\mathrm{A}$ est à $\mathrm{B}$ ce que $\mathrm{C}$ est à ? » est ainsi fournie par un critère de minimisation de complexité (Cornuéjols, 2005).

${ }^{4}$ Cet interdit ne concerne pas les informations qui viennent renforcer le sentiment de véracité de l'histoire.

${ }^{5}$ Cette remarque n'est pas absolue. Par exemple, si le prototype comporte lui-même un aspect spatial, comme les rencontres avec tel collègue qui sont typiquement localisées à proximité immédiate du lieu de travail, l'individualisation de la situation peut comporter un terme $C^{a c c}(s / r)$ non nul.
} 
table que s'il conserve une valeur globalement positive à $U(s)$. Nous allons en voir un exemple.

Dans le cas important où la discrimination de la situation au sein de sa classe s'obtient par la mention d'une caractéristique remarquable $F$, comme dans le cas des religieuses en train de faire un jogging, la formule (18) devient, grâce à (8) et (11) :

$$
U(s)=C^{d i s c r}(s \mid r)-C(F)-\log \left(D_{v}^{r} v_{e}\right)
$$

$D_{v}^{r}$ désigne la densité hypervolumique du prototype. Cette écriture nous permet de comprendre pourquoi un fait remarquable cesse d'être intéressant en devenant ancien. On peut annoncer de but en blanc, comme une nouvelle, qu'une combinaison remarquable vient de sortir au loto, mais on peut plus difficilement le faire si l'événement s'est produit il y a quatorze ans. La configuration du tirage, si elle est structurellement simple, produit un fort contraste entre $C^{d i s c r}(s \mid r)$ et $C(F)$. En revanche, le terme $-\log \left(D_{v}^{r} v_{e}\right)$ est dans ce cas négatif et peut venir considérablement diminuer $U(s)$ (d'environ 10 bits pour quatorze ans à raison de deux tirages de loto par semaine). Noter que ce raisonnement suppose que la dimension temporelle offre l'accès le plus simple à l'événement. Si la conversation porte déjà sur les tirages de loto remarquables, l'accès à l'événement est beaucoup moins complexe, même s'il est ancien, car il se fait dans ce cas au sein de la liste restreinte des tirages mémorables. ${ }^{6}$

Dans le cas d'une narration concernant un fait atypique, les formules (18), (9) et (11) donnent :

$$
U(s)=A(k)-C(F)-\log \left(D_{v}^{r} v_{e}\right)
$$

Le tableau 2 récapitule les différents modes de calcul de l'inattendu $U(s)$.

\footnotetext{
${ }^{6}$ Ce phénomène d'amorçage de complexité offre une explication élégante du fait que les sujets de conversation sont structurellement reliés, ainsi que de l'aversion pour les transitions abruptes.
} 
Définition de l'inattendu.

Partage du calcul de l'inattendu en une part conceptuelle et une part d'accès à la localisation spatiale/temporelle/sociale...

Partage de l'inattendu de $s$ en une part de discrimination par rapport à une référence $r$ et une part d'accès à la localisation de $s$ en tant qu'instance de $r$.

Inattendu conceptuel de la situation $s$ si la caractéristique $F$ la rend unique parmi les instances de la référence $r$.

Inattendu conceptuel d'une situation $s$ présentant une caractéristique $F$ atypique, située à $k$ écarts-types du centre de sa référence.

Inattendu d'accès à la situation $s$ située dans un volume égocentré $v_{e}$, lorsque la densité volumique de ce type d'événement est $D_{v}$.

Inattendu d'accès pour une coïncidence impliquant deux événements similaires inscrits dans le volume $v_{a}$.

Inattendu lorsque la situation $s$ peut être entièrement discriminée des autres instances de la référence $r$ par une caractéristique $F$.

Inattendu lorsque la situation $s$ présente une caractéristique atypique $F$ par rapport à sa référence $r$.

$$
\begin{aligned}
& U(s)=C_{\text {exp }}(s)-C_{o b s}(s) \\
& U(s)=U_{c o n c}(s)+U_{a c c}(s)
\end{aligned}
$$$$
U(s)=U_{d i s c r}(s \mid r)+U_{a c c}(r)
$$

$U_{\text {discr }}(s \mid r)=C^{d i s c r}(s \mid r)-C(F)$

$$
U_{\text {discr }}(s)=A(k)-C(F)
$$

$$
U_{a c c}(s)=-\log \left(D_{v} v_{e}\right)
$$$$
U_{a c c}(s)=-\log \left(D_{v}^{2} v_{e} v_{a}\right)
$$

$$
\begin{aligned}
U(s)= & C^{\text {discr }}(s \mid r)-C(F) \\
& -\log \left(D_{v}^{r} v_{e}\right)
\end{aligned}
$$

$$
\begin{array}{r}
U(s)=A(k)-C(F) \\
-\log \left(D_{v}^{r} v_{e}\right)
\end{array}
$$

Tableau 2. Récapitulation des modes de calcul de l'inattendu cognitif.

Un aspect important du résultat exprimé par (19) est que la complexité de la référence $r$ n'influe pas sur l'intérêt de la narration. Seules comptent :

- la complexité a priori $C^{\text {discr }}(s \mid r)$ liée à la discrimination d'un élément quelconque parmi tous ceux de la classe $r$.

- la complexité $C(F)$ de la caractéristique utilisée pour discriminer $s$.

- la rareté de la référence, mesurée par sa densité hypervolumique $D_{v}^{r}$.

- la proximité de l'événement, mesurée par l'hypervolume égocentré $v_{e}$ qui contient l'événement.

On est en droit de se demander pourquoi les narrations, qui reposent sur une simplicité inattendue, peuvent requérir de nombreux mots pour être rapportées. 
Observons tout d'abord que l'inattendu est un phénomène cognitif, non un phénomène verbal. Il ne se mesure pas en comptant des mots. ${ }^{7}$ Observons ensuite que les formules (19) et (20) ne font pas apparaître la complexité de la référence $r$. La description narrative peut ainsi porter longuement sur $r$ sans nuire à l'intérêt. Il en est de même de tous les détails propres à augmenter la complexité attendue $C_{\text {exp }}$. Ainsi, dans la conversation sur la rencontre inattendue du modèle nu, l'exposé des circonstances : «we went downtown one night to see a movie, and we were sitting in [a restaurant], like downtown, waiting for our movie » se justifie par le fait d'installer une situation dans laquelle l'instanciation des personnes rencontrées est cognitivement complexe. Noter par ailleurs que la formule (6) peut être appliquée de manière récursive, ce qui permet au narrateur de ménager ses effets en distillant les caractéristiques 'incroyables' les unes après les autres. Enfin, comme l'intérêt des narrations repose sur un saut de complexité, l'accumulation de certains détails permet d'augmenter le contraste entre l'attente et l'observation. Tous ces facteurs contribuent à produire des narrations de taille conséquente.

\section{DISCUSSION}

Le modèle que nous venons de présenter présente le grand avantage, si on le compare au modèle antérieur shannonien, de ne reposer que sur des entités accessibles à la cognition des individus engagés dans la conversation, sans requérir aucun accès à des références objectives comme des ensembles exhaustifs d'alternatives (l'univers $\Omega$ des possibles). Les paramètres des formules (19) et (20) sont tous cognitivement plausibles. On pourrait objecter que pour connaître $C^{\text {discr }}(s \mid r)$, il faut connaître l'ensemble des instances possibles couvertes par le prototype $r$. Il n'en est rien : on peut estimer qu'il est complexe de discriminer deux religieuses dans l'ensemble de toutes les religieuses sans connaître cet ensemble. Il suffit d'avoir une estimation du logarithme de son cardinal.

Il est utile de remarquer que, bien que toute utilisation de la notion de probabilité puisse être éliminée des hypothèses du modèle (ce qui nous a permis de définir la probabilité cognitive en (4)), les formules (19) et (20) renferment des aspects de nature statistique. La notion même de prototype repose en partie sur l'accumulation statistique d'expériences similaires. ${ }^{8}$ De plus, la densité hypervolumique $D_{v}^{r}$ renferme elle aussi une expérience accumulée sur la fréquence des événements. ${ }^{9}$ Enfin, l'estimation de $A(k)$ suppose une connaissance, au moins sommaire, de la distribution statistique du prototype selon la caractéristique $F$ considérée. Néanmoins, tant la notion de prototype que l'estimation de la rareté restent des entités cognitivement plausibles, qui sont admissibles dans une modélisation cognitive de la compétence narrative des individus.

\footnotetext{
${ }^{7}$ Nous pensons impossible de développer une théorie prédictive de la compétence narrative qui serait purement comportementale. Les modèles cognitifs s'autorisent à postuler des variables et des processus qui ne sont pas directement observables. C'est ce que nous faisons en définissant la notion de complexité cognitive. De telles hypothèses se justifient par leur capacité de prédiction.

${ }^{8}$ En partie seulement. Les capacités d'induction symboliques, explorées dans des techniques d'intelligence artificielle comme la programmation logique inductive (ILP) ou la généralisation à base d'explications (EBG), sont propres à produire des prototypes en l'absence de toute accumulation statistique.

${ }^{9}$ Là encore, la statistique n'est pas indispensable. Un enfant peut inférer la faible occurrence des incendies à partir d'une expérience unique, en prenant comme estimation de la densité l'inverse de sa durée de vie passée et l'inverse de la distance entre son domicile et le sinistre qui lui est donné d'observer.
} 
Il reste l'estimation de la complexité structurelle, qui intervient notamment dans le terme $C(F)$ ou le terme $C^{\text {conc }}\left(s_{2} \mid s_{1}\right)$ d'une coïncidence à deux termes. Comme nous l'avons suggéré, la complexité de structures peut être estimée à partir d'un modèle psychologique adéquat (e.g. Leyton, 2001), en cumulant la complexité attachée à chaque opération de construction de la structure.

Nous n'avons pas employé le mot pertinence, bien que la présence d'informations dans une narration soit fondamentalement une question liée à leur pertinence. Nous avons évité ce terme pour ne pas introduire de confusion avec la pertinence argumentative, d'une tout autre nature. Cette dernière est généralement la seule qui soit considérée dans les travaux scientifiques. La pertinence narrative, malgré son importance considérable pour la compréhension du fonctionnement cognitif humain, n'a fait l'objet de quasiment aucun travail théorique systématique. ${ }^{10} \mathrm{La}$ 'théorie de la pertinence' (Sperber et Wilson, 1986) se limite, selon nous, à tenter de modéliser la pertinence argumentative et ne dit rien sur la pertinence narrative. Le critère de pertinence qu'elle retient se rapporte à la quantité et à la facilité des inférences. Or si l'on prend les différents termes des formules (19) et (20), aucun n'est prédit par ce critère. Par exemple, la variation graduelle de l'intérêt en fonction de la proximité, représenté par le facteur $v_{e}$, ou de l'atypicité $k$, échappe totalement à tout système inférentiel de nature logique. On notera que la notion de complexité cognitive que nous utilisons n'a à peu près rien à voir avec l'idée de coût cognitif utilisé par Sperber et Wilson. La complexité est évaluée par les individus et entre de manière explicite dans le calcul de l'intérêt. Elle est liée à une difficulté de principe de réaliser l'individualisation d'une situation. Le coût cognitif, quant à lui, est nécessairement implicite et n'est pas évalué par les sujets. Il est lié au fonctionnement effectif de la procédure déductive. La différence ressemble à celle qui existe entre la taille d'un programme fourni à un ordinateur, en nombre d'instructions, et la chaleur additionnelle dégagée par le processeur lorsqu'il exécute ce programme.

\section{Conclusion}

Les résultats exposés ici suggèrent fortement que les êtres humains évaluent la complexité des situations qu'ils perçoivent ou considèrent. La notion de complexité cognitive est liée à la description idéale minimale qui permet d'individualiser une situation, conformément à la notion de complexité introduite dans un cadre mathématique plus général par Andrei Kolmogorov, Gregory Chaitin et Ray Solomonoff dans les années 1960 (Li et Vitanyi, 1993). Nous avons décomposé la complexité cognitive en distinguant la complexité de discrimination, la complexité d'accès et la complexité de structure. La complexité d'accès vise à localiser la situation dans l'espace, dans le temps, dans l'espace social, etc. La complexité de discrimination est associée à l'individualisation de la situation par rapport à un prototype choisi comme référence. La discrimination utilise préférentiellement les caractéristiques atypiques ou exceptionnelles pour rendre la situation observée mentalement non ambiguë. La complexité de structure évalue le caractère 'compressible' (au

\footnotetext{
${ }^{10}$ Citons tout de même, dans un domaine connexe qui est celui de la littérature, le travail de Patrick Hogan (Hogan, 2003) qui, en recherchant les universaux de la narration littéraire, touche à ces questions de pertinence narrative. Le travail de Neal Norrick sur la conversation (Norrick, 2000) aborde également ces questions, quoique souvent de manière implicite.
} 
sens informatique) de la situation. Elle diminue en présence de symétries, de duplications et plus généralement de formes simples.

La complexité cognitive intervient dans de nombreux aspects très importants de la cognition humaine (Chater \& Vitányi, 2003). Nous avons évoqué ici son importance dans la détermination de l'intérêt, que nous pouvons résumer ainsi : Toute situation qui provoque une diminution inattendue de la complexité cognitive est perçue comme intéressante et est susceptible d'être communiquée sur le mode narratif. L'adjectif 'inattendu' traduit la différence, exprimée dans la formule (3), entre la complexité attendue et la complexité observée.

Nous avons souligné quelques aspects fortement prédictifs de cette loi, en particulier l'influence de la proximité, l'impact des coïncidences et l'importance de choisir des caractérisations simples. Ces résultats étaient en partie inaccessibles à la théorie antérieure probabiliste, et totalement inaccessibles aux théories inférentielles.

La caractérisation de l'intérêt que nous avons obtenue est une caractérisation suffisante, mais partielle. La diminution inattendue de complexité se combine à un autre facteur dont nous n'avons pas parlé ici, qui est le changement d'intensité émotionnelle (Dessalles, 2007). Ainsi, la nouvelle d'une naissance pourra avoir un intérêt considérable pour les proches, même si elle n'est pas inattendue. ${ }^{11}$ Certains pourraient voir dans la surprise liée à l'inattendu une forme particulière de changement émotionnel (Reisenzein, 2000). Cette assimilation n'apparaît cependant pas souhaitable, en raison du processus particulier, fondé sur la complexité cognitive, qui conduit à la détection des situations inattendues. Inversement, les émotions qui entrent dans la détermination de l'intérêt requièrent de la part des interlocuteurs un certain niveau d'empathie avec les personnes impliquées dans la situation rapportée, ce qui n'est pas le cas de la surprise associée à un saut de complexité (penser à l'exemple du tirage de loto consécutif).

Nous avons seulement commencé ici l'exploration des conséquences de la sensibilité humaine à la complexité. Nous avons étudié certains aspects de la détermination de l'intérêt narratif, sans en tirer toutes les conséquences, notamment en ce qui concerne bon nombre de motivations humaines liées à la vie quotidienne. Nous sommes convaincu qu'il s'agit là d'une voie de recherche potentiellement féconde.

\section{RÉFÉRENCES}

Bar-Hillel, M. (1980). The base-rate fallacy in probability judgments. Acta Psychologica, 44, 3, 211-233.

Behne, T., Carpenter M. \& et al. (2005). Unwilling versus unable: infants' understanding of intentional action. Developmental psychology, 41, 2, 328-337. www.eva.mpg.de/psycho/staff/carpenter/pdf/Behne_et_al2005_DP.pdf

Berkowitz, D. (1990). Refining the gatekeeping metaphor for local television news. Journal of Broadcasting \& Electronic Media, 34 ,1, 55-68.

Brown, R. \& Kulik J. (1977). Flashbulb memories. Cognition 5, 73-99.

Call, J., Hare B. \& Tomasello M. (1998). Chimpanzee gaze following in an object choice task. Animal Cognition, 1, 89-99.

\footnotetext{
${ }^{11}$ L'analyse du changement émotionnel laisse également apparaître des dépendances logarithmiques, cette fois en fonction de stimulus comme un nombre de victimes, une somme d'argent gagnée, la durée d'une condamnation. Le changement émotionnel se combine à l'inattendu, par exemple pour donner la loi du mort-kilomètre, bien connue dans les écoles de journalisme : pour susciter le même intérêt, un drame doit faire d'autant plus de victimes qu'il s'est produit en un lieu éloigné (Dessalles, 2005).
} 
Carpenter, M., Nagell K. \& Tomasello M. (1998). Social cognition, joint attention, and communicative competence from 9 to 15 months of age. Monographs of the Society for Research in Child Development, 255, 63, 1-143.

Chater, N. \& Vitányi, P. (2003). Simplicity: a unifying principle in cognitive science? Trends in cognitive sciences, 7, 1, 19-22.

Cornuéjols, A. (1996). Analogie, principe d'économie et complexité algorithmique. Actes des 1lèmes Journées Françaises de l'Apprentissage. Sète. www.lri.fr/ antoine/Papers/JFA96-final-osX.pdf

Csányi, G. \& Szendröi B. (2004). Fractal-small-world dichotomy in real-world networks. Physical Review E, 70, 1. www.csanyi.net/Publications/scaleproof.pdf

Dessalles, J-L. (2002). La fonction shannonienne du langage : un indice de son évolution. Langages, 146, 101-111. www.enst.fr/ jld/papiers/pap.evol/Dessalles_01040903.pdf

Dessalles, J-L. (2005). Vers une modélisation de l'intérêt. In A. Herzig, Y. Lespérance \& A-I. Mouaddib (éds), Actes des troisièmes journées francophones 'Modèles formels de l'interaction' (MFI-05) (pp. 113-122). Toulouse: Cépaduès Éditions. www.enst.fr/ jld/papiers/pap.conv/Dessalles_04122102.pdf

Dessalles, J-L. (2006a). A structural model of intuitive probability. In D. Fum, F. Del Missier \& A. Stocco (éds), Proceedings of the seventh International Conference on Cognitive Modeling (pp. 86-91). Trieste, IT: Edizioni Goliardiche. www.enst.fr/ jld/papiers/pap.cogni/Dessalles_06020601.pdf

Dessalles, J-L. (2006b). Trivialization behaviour in conversation. In Proceedings of the 2nd Conference on Language, Culture and Mind (pp. 44-46). Paris: École Nationale Supérieure des Télécommunications. www.enst.fr/ jld/papiers/pap.conv/Dessalles_06072303.pdf

Dessalles, J-L. (2007). Le rôle de l'impact émotionnel dans la communication des événements. In J. Lang, Y. Lespérance, D. Sadek \& N. Maudet (éds), Actes des journées francophones 'Modèles formels de l'interaction' (MFI-07) (pp. 113-125). Paris: Annales du LAMSADE, Université Paris Dauphine. www.enst.fr/ jld/papiers/pap.conv/Dessalles_07022101.pdf

Dunbar, R. I. M. (1998). Theory of mind and the evolution of language. In J. R. Hurford, M. Studdert-Kennedy \& C. Knight (éds), Approaches to the evolution of language: social and cognitive bases (pp. 92-110). Cambridge: Cambridge University Press.

Eggins, S. \& Slade D. (1997). Analysing casual conversation. London: Equinox.

Galtung, J. \& Ruge M. H. (1965). The structure of foreign news: The presentation of the Congo, Cuba and Cyprus crises in four foreign newspapers. Journal of International Peace Research, 1, 64-90.

Hogan, P. C. (2003). The mind and its stories - Narrative universals and human emotion. Cambridge, UK: Cambridge University Press.

Itti, L. \& Koch C. (2001). Computational modelling of visual attention. Nature Reviews Neuroscience, 195-203. ilab.usc.edu/publications/doc/Itti_Koch01nrn.pdf

Kahneman, D. \& Tversky A. (1982). Subjective probability: A judgement of representativeness. Cognitive Psychology, 3, 430-454.

Landragin, F. (2004). Saillance physique et saillance cognitive. Corela, 2, 2. edel.univ-poitiers.fr/corela/document.php?id=142

Leyton, M. (1992). Symmetry, causality, mind. Cambridge MA: The MIT Press.

Leyton, M. (2001). A generative theory of shape. New York: Springer Verlag, 2145.

Li, M. \& Vitanyi P. (1993). An introduction to Kolmogorov complexity and its applications. New York: Springer Verlag (édition 1997).

Liszkowski, U., Carpenter M., T. Striano \& M. Tomasello (2004). Twelve-month-olds point to share attention and interest. Developmental science, 7, 3, 297-307. www.eva.mpg.de/psycho/pdf/twelve-month-olds.pdf

MacDougall, C. D. \& Reid R. D. (1957). Interpretative reporting. New York: Macmillan (édition 1987).

Maclean, M. \& Pinna L. (1958). Distance and news interest: Scarperia, Italy. Journalism Quarterly, 35, 36-48.

Nisbett, R. E. \& Ross L. (1980). Human inference: strategies and shortcomings of social judgments. Englewood Cliffs, N. J: Prentice Hall. 
Norrick, N. R. (2000). Conversational narrative: storytelling in everyday talk. Amsterdam: John Benjamins Publishing Company.

Reisenzein, R. (2000). The subjective experience of surprise. In H. Bless \& J. P. Forgas (éds), Subjective experience in social cognition and social behaviour (p. 262-279). Philadelphia, PA: Psychology Press.

Savoie, D. \& Ladouceur R. (1995). Évaluation et modification de conceptions erronées au sujet des loteries. Canadian Journal of Behavioural Science, 27, 2, 199-213.

Shannon, C. E. (1948). A mathematical theory of communication. The Bell System Technical Journal, 27, 379-423,623-656.

Shapiro, M. A. \& Fox J. R. (2002). The role of typical and atypical events in story memory. Human Communication Research, 28, 1, 109-135.

Solomonoff, R. J. (1997). The discovery of algorithmic probability. Journal of Computer and System Sciences, 55, 1, 73-88. http://www.cwi.nl/ paulv/papers/solomonoff.ps

Sperber, D. \& Wilson D. (1986). La pertinence. Paris: Les Editions de Minuit (édition 1989).

Stangor, C. \& McMillan D. (1992). Memory for expectancy-congruent and expectancyincongruent information: a review of the social and social developmental literatures. Psychological Bulletin, 111, 1, 42-61.

Tannen, D. (1984). Conversational Style - Analyzing talk among friends. Norwood: Ablex Publishing Corporation.

Tannen, D. (1989). Talking voices - Repetition, dialogue, and imagery in conversational discourse. Cambridge, UK: Cambridge university press.

Terrell, D. (1994). A test of the gambler's fallacy: Evidence from pari-mutuel games. Journal of risk and uncertainty, 8, 3, 309 - 317.

Tversky, A. \& Kahneman D. (1983). Extensional versus intuitive reasoning: The conjunction fallacy in probability judgment. Psychological Review, 90, 4, 293-315.

Warren, C. N. (1934). Modern news reporting. New York: Harper \& Brothers (édition 1959).

Woll, S. \& Graesser A. (1982). Memory discrimination for information typical and atypical of person schemata. Social Cognition, 1, 287-310. 